\title{
Sulfonium-based Ionic Liquids Incorporating the Allyl Functionality
}

\author{
Dongbin Zhao, Zhaofu Fei, Wee Han Ang and Paul J. Dyson* \\ Institut des Sciences et Ingénierie Chimiques, Ecole Polytechnique Fédérale de Lausanne (EPFL), \\ CH-1015 Lausanne, Switzerland. Email: paul.dyson@epfl.ch \\ * Author to whom correspondence should be addressed: Email: paul.dyson @epfl.ch, Fax: 0041-21- \\ 693-9885
}

Received: 23 March 2007 / Accepted: 10 April 2007 / Published: 16 April 2007

\begin{abstract}
A series of sulfonium halides bearing allyl groups have been prepared and characterized. Anion metathesis with $\mathrm{Li}\left[\mathrm{Tf}_{2} \mathrm{~N}\right]$ and $\mathrm{Ag}\left[\mathrm{N}(\mathrm{CN})_{2}\right]$ resulted in sulfonium-based ionic liquids which exhibit low viscosities at room temperature. The solid state structure of one of the halide salts was determined by single crystal X-ray diffraction.
\end{abstract}

Keywords: Sulfonium salts, ionic liquids, allyl group, viscosity, X-ray structure.

\section{Introduction}

Ionic liquids containing imidazolium cations have been extensively studied, and have found applications in catalysis [1], analytical chemistry [2], and elsewhere [3]. The development of functionalized (or task-specific) ionic liquids received much attention due to the potential to impart the liquid with specific chemical and/or physical properties [3,4]. Various functional groups have been incorporated onto imidazolium [5], ammonium [6], pyridinium [7], triazolium [8], oxazolidinium and morpholinium [9], pyrazinium, and pyrimidinium cations [10]. In addition, ionic liquids with functionalized anions have also been reported [11]. In contrast, sulfonium-based ionic liquids have received less attention possibly because the resulting sulfonium salts are generally less stable than their imidazolium counterparts. However, sulfonium salts have certain useful properties that could be applied in specialised areas. For example, a series of trialkylsulfonium iodides were evaluated as electrolytes in dye-sensitized solar cells [12]. Although the energy conversion efficiencies were low relative to systems based on imidazolium electrolytes [13], doping the sulfonium-based electrolytes 
with metal iodides such as CuI, AgI or LiI resulted in improved conversion efficiencies [14]. Sulfonium salts also represent a source of sulfonium ylides that can be generated in situ as synthons for chiral compounds [15].

A more recent report indicated that trialkylsulfonium salts based on the dicyanamide anion have low viscosities compared to other ionic liquids, typically in the range $20-60 \mathrm{cP}$ at $20^{\circ} \mathrm{C}$, which compares very favourably to imidazolium dicyanamides [16]. Viscosity is an extremely important parameter in many potential applications of ionic liquids including their use as electrolytes in solar cells [17, 18, 19]. It has been shown that the viscosity of the imidazolium based ionic liquids can be reduced using asymmetric anions [11], and in addition, it has been found that in certain cases allyl groups attached to imidazolium cations resulted in low viscosity ionic liquids in combination with iodide [20, 21], the preferred anion in dye sensitized solar cells. Thus, we thought it would be interesting to incorporate the allyl group onto the sulfonium cation in order to see if the viscosities of the resulting ionic liquids could be further reduced.

\section{Results and Discussion}

The synthetic route used to prepare the allyl-functionalized sulfonium salts described herein is illustrated in Scheme 1. In the first step the appropriate allyl halide and disulphide are reacted in acetone and stirred at room temperature to afford dialkyl-allyl-sulfonium halides, $\left[\mathrm{C}_{2}\right.$ allylsul]Br $\mathbf{1}$ and [C $\mathrm{C}_{4}$ allylsul]I 2. Diethyl sulphide reacts rapidly with allyl bromide to afford product $\mathbf{1}$ as a colourless powder directly from the reaction mixture. In contrast, the synthesis of $\mathbf{2}$ required the more active alkylation agent, allyl iodide, in order to proceed under mild conditions and in high yield.

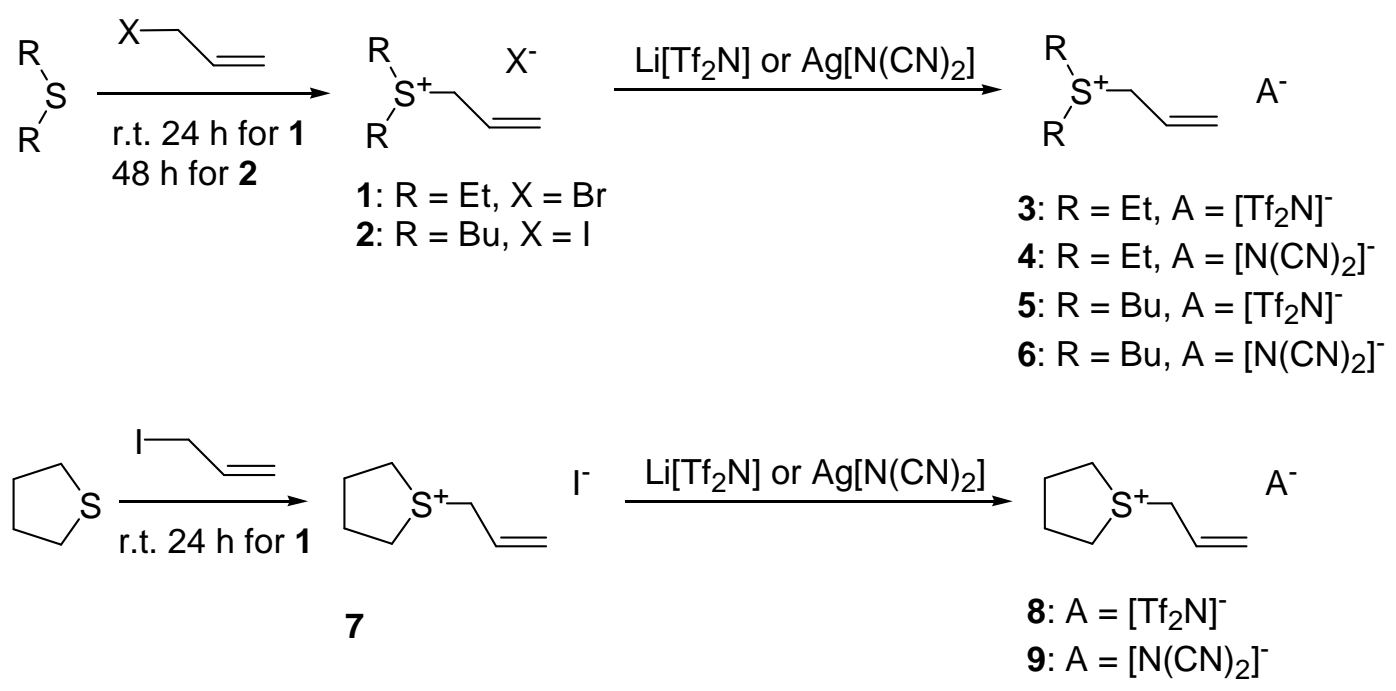

Scheme 1. Synthesis of sulfonium-based ionic liquids.

Subsequent metathesis of $\mathbf{1}$ and $\mathbf{2}$ in water with lithium bis(trifluoromethanesulfonyl)imide $\left(\operatorname{LiTf}_{2} \mathrm{~N}\right)$ or silver dicyanamide $\left(\mathrm{Ag}\left[\mathrm{N}(\mathrm{CN})_{2}\right]\right)$, freshly prepared by reacting $\mathrm{AgNO}_{3}$ and $\mathrm{Na}\left[\mathrm{N}(\mathrm{CN})_{2}\right]$ in equimolar quantities, afford the corresponding bis(trifluoromethanesulfonyl)imide (3 and $\mathbf{5}$ ) and dicyanamide (4 and 6) salts, which are all liquids at room temperature. It should be noted that 
dimethylallyl and diallylmethyl sulfonium cations have been reported previously although their physical properties were not described in detail [22]. Following a similar route sulfonium salts based on tetrahydrothiophene, [allylthio]I 7, [allylthio] $\mathrm{Tf}_{2} \mathrm{~N} 8$ and [allylthio] $\mathrm{N}(\mathrm{CN})_{2} 9$ were prepared.

Complexes 1 - 9 were characterised using electrospray ionisation mass spectrometry (ESI-MS), IR, ${ }^{1} \mathrm{H}$ and ${ }^{13} \mathrm{C}$ NMR spectroscopy. They were found to be spectroscopically pure by NMR and their purity was further confirmed using elemental analyses. The positive ion ESI mass spectra of $\mathbf{1}$ - 9 exhibit parent peaks at $\mathrm{m} / \mathrm{z} 131,201$ and 97 corresponding to the cations $\left[\mathrm{C}_{2} \text { allylsul }\right]^{+},\left[\mathrm{C}_{4} \text { allylsul }\right]^{+}$and [allylthio] $^{+}$, respectively. In negative ion mode intense peaks corresponding to the anticipated anions were observed. In keeping with previous observations [23], aggregates based on small cation-anion clusters are observed, which are reduced in relative intensity as the concentration of the ionic liquid solution is reduced in the co-solvent. The main feature in the IR spectra of $\mathbf{1}-\mathbf{9}$ is the characteristic $\mathrm{C}=\mathrm{C}$ vibrations observed in the range $1635-1638 \mathrm{~cm}^{-1}$. The ${ }^{1} \mathrm{H}$ and ${ }^{13} \mathrm{C}$ NMR spectra of $1-9$ in $\mathrm{D}_{2} \mathrm{O}$ or $\mathrm{CD}_{3} \mathrm{CN}$ are as expected with little change in the spectra as the anion is varied and are routine in nature.
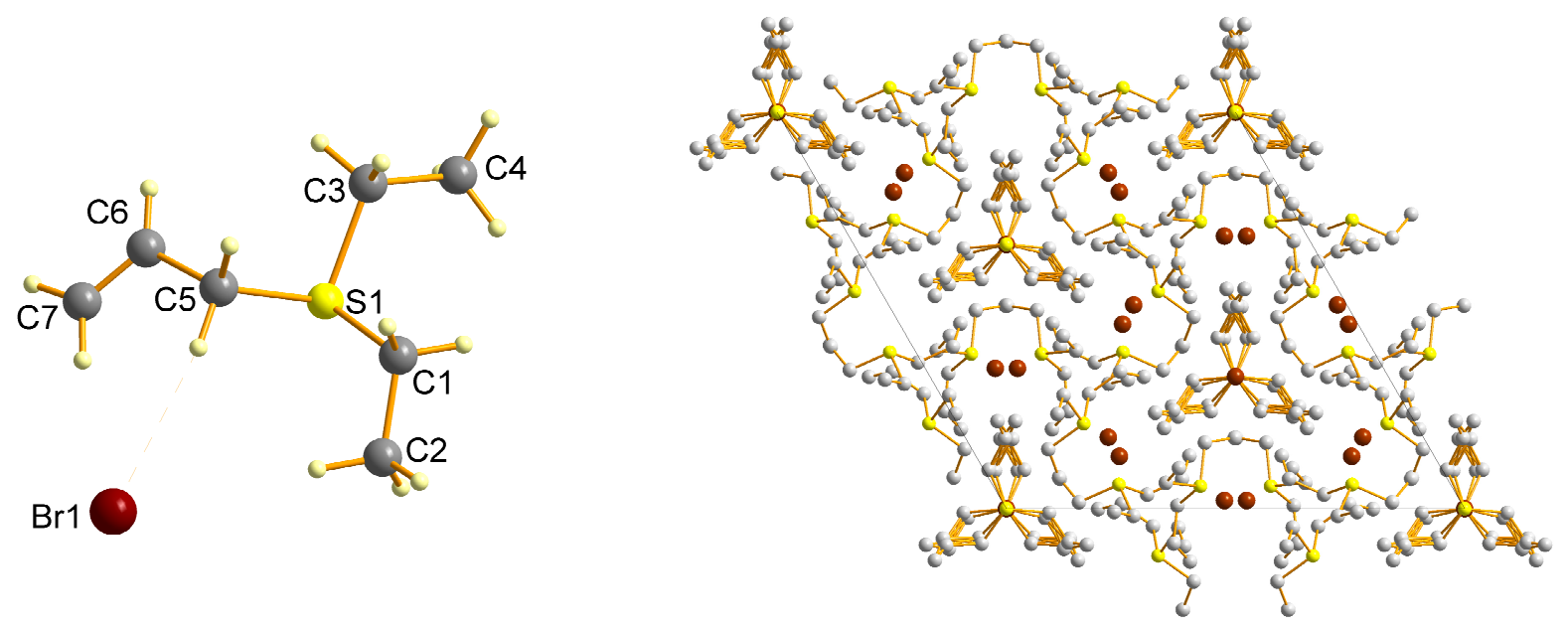

Figure 1. Ball and stick representation of 1 (left); disordered atoms omitted for clarity. Packing diagram of 1 (right) view along the $c$-axis. Key bond lengths $(\AA): \mathrm{S}-\mathrm{C}_{\mathrm{ave}}, 1.814 ;\left(s p^{3}\right) \mathrm{C}-\left(s p^{3}\right) \mathrm{C}_{\text {ave }}$,

$$
1.545 ;\left(s p^{3}\right) \mathrm{C}-\left(s p^{2}\right) \mathrm{C}_{\mathrm{ave}}, 1.529 ;\left(s p^{2}\right) \mathrm{C}-\left(s p^{2}\right) \mathrm{C}_{\mathrm{ave}}, 1.292 \text {. }
$$

Single crystals of $\mathbf{1}$ suitable for X-ray diffraction analysis were grown from acetonitrile solution by slow diffusion with diethyl ether. The structure of $\mathbf{1}$ is shown in Figure 1 and key bond parameters are given in the caption. The compound crystallised in the trigonal lattice system as disordered molecules, presumably due to the highly flexible ethyl and allyl groups. In addition, one of the molecules in the asymmetric cells lies on a special positionsymmetry element and it is not possible to determine the exact orientation of that molecule. Thus, it is depicted as the disordered rosette structure shown in the packing diagram in Figure 1. Numerous weak H-bonding interactions were also observed between the aliphatic $\mathrm{H}$-atoms and the bromide atoms at distances of 2.78-2.93 $\AA$. Similar interactions were also found in polyalkylammonium bromide compounds such as diethyl-di- $n$-propylammonium bromide and tetraethylammonium bromide [24]. 
With the exception of $\mathbf{1}$ all the allyl-functionalized sulfonium salts are liquid at room temperature, most with a glass transition temperature $\left(\mathrm{T}_{\mathrm{g}}\right)$ of approximately $-60^{\circ} \mathrm{C}$ (see Table 1 ). In general, the viscosities of the sulfonium ionic liquids (except for the halide salts) are low, and as expected, dependent on the nature of the anion. It is noteworthy that while allyl functionalized imidazolium ionic liquids have lower viscosity than the saturated alkyl congeners [21, 25]; in the case the sulfonium salts, introduction of the allyl functionality does not result in lower viscosities $[12,16]$. In our previous paper [21], we hypothesized that the reduced viscosity of the allyl-substituted imidazolium salts may be due to a decrease in hydrogen bonding between the imidazolium cation and the anion at the expense of increased $\pi-\pi$ interactions. Since there are no acidic protons in the sulfonium salts described herein the incorporation of alkyl groups does not reduce their viscosity, further validating the earlier hypothesis. In fact, it was observed that the allyl-sulfonium ionic liquids described herein have slightly higher viscosities compared to related allyl-based systems. Presumably the slight increase in viscosity is due to the presence of additional weak $\pi-\pi$ interactions in the liquid phase. While such qualitative ideas require substantiation they could prove useful in developing theoretical methods to predict the physical properties of ionic liquids [26]. The higher viscosity of the ionic liquids with the cyclic sulfonium cation ( 8 and $\mathbf{9}$ ) compared to $\mathbf{3}$ and $\mathbf{4}$ could be due to reduced conformational flexibility and such phenomena have previously been observed in cyclic quaternary ammonium ionic liquids [27].

Table 1. Physical properties of allyl-sulfonium ionic liquids.

\begin{tabular}{lccc}
\hline Ionic liquids & $T_{g}\left({ }^{\circ} \mathrm{C}\right)$ & $T_{m}\left({ }^{\circ} \mathrm{C}\right)$ & $\eta\left(\mathrm{cP}, 20^{\circ} \mathrm{C}\right)$ \\
\hline$\left[\mathrm{C}_{4}\right.$ Allylsul][ 2 & -60.12 & - & 1080.0 \\
{$\left[\mathrm{C}_{2}\right.$ Allylsul] $\left[\mathrm{Tf}_{2} \mathrm{~N}\right] \mathbf{3}$} & - & -60.19 & 42.6 \\
{$\left[\mathrm{C}_{2}\right.$ Allylsul] $\left[\mathrm{N}(\mathrm{CN})_{2}\right] \mathbf{4}$} & -42.34 & - & 31.0 \\
{$\left[\mathrm{C}_{4}\right.$ Allylsul] $\left[\mathrm{Tf} \mathrm{f}_{2} \mathrm{~N}\right] \mathbf{5}$} & -58.15 & - & 108.0 \\
{$\left[\mathrm{C}_{4}\right.$ Allylsul] $\left[\mathrm{N}(\mathrm{CN})_{2}\right] \mathbf{6}$} & -42.34 & - & 89.5 \\
{$[$ Allylthio][ 7 } & -41.11 & - & 1283.0 \\
{$\left[\right.$ Allylthio] $\left[\mathrm{Tf}{ }_{2} \mathrm{~N}\right] \mathbf{8}$} & -52.62 & - & 80.0 \\
{$\left[\right.$ Allylthio] $\left[\mathrm{N}(\mathrm{CN})_{2}\right] \mathbf{9}$} & -83.36 & - & 66.5 \\
\hline
\end{tabular}

Concluding comments: We have, as far as we are aware, described the first examples of functionalized sulfonium-based ionic liquids. Unlike imidazolium salts, the presence of the allyl group does not reduce the viscosity of the resulting ionic liquids, presumably because the allyl group helps to reduce hydrogen bonding interactions in the imidazolium-based ionic liquids, albeit at the expense of increase $\pi-\pi$ interactions. The flexibility of the cation also appears to play an important role in the physical properties of the ionic liquids. The ionic liquids could prove to be useful in various applications such as electrolytes for dye sensitized solar cells. 


\section{Materials and Methods}

All reagents were obtained commercially and used as received. The synthesis of the 1, 2 and $\mathbf{7}$ was performed under an inert atmosphere of dry nitrogen using standard Schlenk techniques in solvents dried and distilled prior to use. All other compounds were made without precautions to exclude air or moisture. IR spectra were recorded on a Perkin-Elmer FT-IR 2000 system. NMR spectra were measured at $20^{\circ} \mathrm{C}$ on a Bruker DMX 400, using $\mathrm{SiMe}_{4}$ as external standard. Electrospray ionization mass spectra (ESI-MS) were recorded on a ThermoFinnigan LCQ ${ }^{\mathrm{TM}}$ Deca XP Plus quadrupole ion trap instrument on samples diluted (methanol, ethanol, water or DMSO). Samples were infused directly into the source at $5 \mu \mathrm{L} \mathrm{min}^{-1}$ using a syringe pump and the spray voltage was set at $5 \mathrm{kV}$ and the capillary temperature at $50^{\circ} \mathrm{C}$ [28]. Thioethers should be handled with special caution due to their highly unpleasant odours. The reaction waste was treated with $\mathrm{H}_{2} \mathrm{O}_{2}(10 \%$ v/v).

\section{Synthesis of [ $C_{2}$ Allylsul $] \mathrm{Br} \mathbf{1}$}

A solution of diethyl sulphide $(9.02 \mathrm{~g}, 0.10 \mathrm{~mol})$ and allyl bromide $(12.1 \mathrm{~g}, 0.10 \mathrm{~mol})$ in acetone (20 $\mathrm{ml}$ ) was stirred at r.t. for $24 \mathrm{~h}$ during which time a white precipitate formed. The precipitate was removed, washed with diethyl ether $(3 \times 30 \mathrm{ml})$ and dried under vacuum for $24 \mathrm{~h}$, yield: $20.6 \mathrm{~g}$, 98\%; M.p. $145^{\circ}$ C. Crystals suitable for X-ray diffraction were obtained by slow diffusion of diethyl ether into an acetonitrile solution of the compound at r.t. ESI-MS $\left(\mathrm{H}_{2} \mathrm{O}\right)$ : positive ion: $131\left[\mathrm{C}_{2} \mathrm{Allylsul}\right.$, negative ion: $80[\mathrm{Br}] .{ }^{1} \mathrm{H}$ NMR $\left(\mathrm{D}_{2} \mathrm{O}\right): \delta=5.88(\mathrm{~m}, 1 \mathrm{H}), 5.68(\mathrm{~s}, 1 \mathrm{H}), 5.65(\mathrm{~s}, 1 \mathrm{H}), 4.03(\mathrm{~m}, 2 \mathrm{H}), 3.29$ (tetra, $4 \mathrm{H}), 1.43$ (triple $6 \mathrm{H}) ;{ }^{13} \mathrm{C}$ NMR $\left(\mathrm{D}_{2} \mathrm{O}\right): \delta=127.6,123.3,39.9,32.5,8.6$; IR $\left(\mathrm{cm}^{-1}\right): 2971,2935$, 2903, 2832 ( $v_{\mathrm{C}-\mathrm{H}}$ alkane), $1638\left(v_{\mathrm{C}=\mathrm{C}}\right.$ alkene); Anal. Calcd for $\mathrm{C}_{7} \mathrm{H}_{15} \mathrm{BrS}(\%)$ : C, 39.82; H, 7.16; Found: C, 39.87; H, 7.18.

\section{Synthesis of [C $C_{4}$ Allylsul]I 2}

A solution of dibutyl sulphide (14.6 g, $0.10 \mathrm{~mol})$ and allyl iodide $(16.8 \mathrm{~g}, 0.10 \mathrm{~mol})$ in acetone (20 $\mathrm{ml}$ ) was stirred at r.t. for $48 \mathrm{~h}$, during which time a pale yellow liquid phase formed. The acetone was removed and the remaining liquid was washed with diethyl ether $(3 \times 30 \mathrm{ml})$ and dried under vacuum for $24 \mathrm{~h}$, yield: $17.9 \mathrm{~g}, 57 \%$; ESI-MS $\left(\mathrm{H}_{2} \mathrm{O}\right)$ : positive ion: 201 [C $\mathrm{C}_{4}$ Allylsul], negative ion: 127 [I]. ${ }^{1} \mathrm{H}$ NMR $\left(\mathrm{D}_{2} \mathrm{O}\right): \delta=5.89(\mathrm{~m}, 1 \mathrm{H}), 5.69(\mathrm{~s}, 1 \mathrm{H}), 5.66(\mathrm{~s}, 1 \mathrm{H}), 3.95(\mathrm{~m}, 2 \mathrm{H}), 3.16$ (triple, $\left.4 \mathrm{H}\right), 1.78$ (penta $4 \mathrm{H}) ; 1.50$ (penta 4H), 0.98 (triple, $6 \mathrm{H}){ }^{13} \mathrm{C} \mathrm{NMR}\left(\mathrm{D}_{2} \mathrm{O}\right): \delta=127.6,123.5,40.8,38.1,25.6,21.2,12.6$; IR $\left(\mathrm{cm}^{-1}\right)$ : 2968, 2939, 2879, ( $\mathrm{v}_{\mathrm{C}-\mathrm{H}}$ alkane), 1638 ( $\mathrm{v}_{\mathrm{C}=\mathrm{C}}$ alkene); Anal. Calcd for $\mathrm{C}_{11} \mathrm{H}_{23} \mathrm{IS}(\%)$ : C, 42.04; H, 7.38; Found: C, 41.95, H, 7.41.

\section{Synthesis of $\left[\mathrm{C}_{2}\right.$ Allylsul] $\left[\mathrm{Tf}_{2} \mathrm{~N}\right] \mathrm{3}$}

A solution of $1(10.5 \mathrm{~g}, 0.05 \mathrm{~mol})$ and $\mathrm{Li}\left[\mathrm{Tf}_{2} \mathrm{~N}\right](13.4 \mathrm{~g}, 0.05 \mathrm{~mol})$ in water $(20 \mathrm{ml})$ was stirred at r.t. for $1 \mathrm{~h}$, during which time a second liquid phase formed. The lower phase was removed, washed with water $(3 \times 20 \mathrm{ml})$ and dried under vacuum for $24 \mathrm{~h}$, yield: $16 \mathrm{~g}, 78 \%$; ESI-MS (Ethanol): positive ion: 
131 [ $\mathrm{C}_{2}$ Allylsul], negative ion: $280\left[\mathrm{Tf}_{2} \mathrm{~N}\right] .{ }^{1} \mathrm{H}$ NMR $\left(\mathrm{D}_{2} \mathrm{O}\right): \delta=5.87(\mathrm{~m}, 1 \mathrm{H}), 5.66(\mathrm{~s}, 1 \mathrm{H}), 5.64(\mathrm{~s}$, $1 \mathrm{H}), 4.03(\mathrm{~m}, 2 \mathrm{H}), 3.24$ (tetra, $4 \mathrm{H}), 1.47$ (triple $6 \mathrm{H}) ;{ }^{13} \mathrm{C}$ NMR $\left(\mathrm{D}_{2} \mathrm{O}\right): \delta=127.2,123.4,121.0,40.1$, 32.6, 8.2; IR $\left(\mathrm{cm}^{-1}\right): 2961,2935,2905,2839\left(v_{\mathrm{C}-\mathrm{H}}\right.$ alkane), $1637\left(v_{\mathrm{C}=\mathrm{C}}\right.$ alkene); Anal. Calcd for $\mathrm{C}_{9} \mathrm{H}_{15} \mathrm{~F}_{6} \mathrm{NO}_{4} \mathrm{~S}_{3}(\%)$ : C, 26.27; H, 3.67; N, 3.40; Found: C, 26.27; H, 3.61; N, 3.38.

\section{Synthesis of $\left[\mathrm{C}_{2} \mathrm{Allylsul}\right]\left[\mathrm{N}(\mathrm{CN})_{2}\right] \mathbf{4}$}

Silver dicyanamide was prepared by mixing silver nitrate and sodium dicyanamide in aqueous solution followed by filtration. A solution of $1(10.5 \mathrm{~g}, 0.05 \mathrm{~mol})$ and $\mathrm{Ag}\left[\mathrm{N}(\mathrm{CN})_{2}\right](8.5 \mathrm{~g}, 0.05 \mathrm{~mol})$ in water $(20 \mathrm{ml})$ was stirred at $80^{\circ} \mathrm{C}$ for $1 \mathrm{~h}$, the mixture was filtered and the filtrate was dried under vacuum. The colourless liquid was then washed with diethyl ether $(3 \times 20 \mathrm{ml})$ and dried under vacuum for $24 \mathrm{~h}$, yield: $8.7 \mathrm{~g}, 89 \%$; ESI-MS (Ethanol): positive ion: 131 [ $\mathrm{C}_{2}$ Allylsul], negative ion: 66 $\left[\mathrm{N}(\mathrm{CN})_{2}\right] .{ }^{1} \mathrm{H}$ NMR $\left(\mathrm{D}_{2} \mathrm{O}\right): \delta=6.12(\mathrm{~m}, 1 \mathrm{H}), 5.83(\mathrm{~s}, 1 \mathrm{H}), 5.88(\mathrm{~s}, 1 \mathrm{H}), 4.70(\mathrm{~m}, 2 \mathrm{H}), 3.43$ (tetra, 4H), 1.67 (triple 6H); ${ }^{13} \mathrm{C}$ NMR $\left(\mathrm{D}_{2} \mathrm{O}\right): \delta=127.8,123.4,120.0,40.2,32.7,8.6$; IR $\left(\mathrm{cm}^{-1}\right): 2978,2938,\left(\mathrm{v}_{\mathrm{C}-\mathrm{H}}\right.$ alkane), 1638 ( $v_{\mathrm{C}=\mathrm{C}}$ alkene); Anal. Calcd for $\mathrm{C}_{11} \mathrm{H}_{17} \mathrm{~N}_{3} \mathrm{~S}(\%)$ : C, 59.16; H, 7.68; N, 18.83; Found: C, 59.17; H, 7.71; N, 18.79 .

\section{Synthesis of [C $\mathrm{C}_{4}$ Allylsul] $\left[\mathrm{Tf}_{2} \mathrm{~N}\right] \mathbf{5}$}

A solution of $2(16.4 \mathrm{~g}, 0.05 \mathrm{~mol})$ and $\mathrm{Li}\left[\mathrm{Tf}_{2} \mathrm{~N}\right](13.4 \mathrm{~g}, 0.05 \mathrm{~mol})$ in water $(20 \mathrm{ml})$ was stirred at r.t. for $1 \mathrm{~h}$, during which time a second liquid phase formed. The ionic liquid phase was removed, washed with water $(3 \times 20 \mathrm{ml})$ and dried under vacuum for $24 \mathrm{~h}$, yield: $18.5 \mathrm{~g}, 77 \%$; ESI-MS $\left(\mathrm{H}_{2} \mathrm{O}\right)$ : positive ion: 201 [C $\mathrm{C}_{4}$ Allylsul], negative ion: $280\left[\mathrm{Tf}_{2} \mathrm{~N}\right] .{ }^{1} \mathrm{H}$ NMR $\left(\mathrm{D}_{2} \mathrm{O}\right): \delta=5.91(\mathrm{~m}, 1 \mathrm{H}), 5.64(\mathrm{~s}, 1 \mathrm{H}), 5.60$ (s, 1H), 3.94 (m, 2H), 3.12 (triple, $4 \mathrm{H}$ ), 1.79 (penta 4H); 1.49 (penta 4H), 0.99 (triple, $6 \mathrm{H}){ }^{13} \mathrm{C} \mathrm{NMR}$ $\left(\mathrm{D}_{2} \mathrm{O}\right): \delta=127.6,123.5,117.3,40.8,38.1,25.6,21.2,12.7 ; \mathrm{IR}\left(\mathrm{cm}^{-1}\right): 2969,2942,2870$, (ve-H alkane), 1638 ( $v_{\mathrm{C}=\mathrm{C}}$ alkene); Anal. Calcd for $\mathrm{C}_{14} \mathrm{H}_{25} \mathrm{~F}_{6} \mathrm{NO}_{4} \mathrm{~S}_{3}(\%)$ : C, 34.92; H, 5.23; N, 2.91; Found: C, 34.95, H, 5.28, N, 2.87.

\section{Synthesis of $\left[\mathrm{C}_{4} \mathrm{Allyl}\right.$ sul $]\left[\mathrm{N}(\mathrm{CN})_{2}\right] 6$}

A solution of $2(16.4 \mathrm{~g}, 0.05 \mathrm{~mol})$ and $\mathrm{Ag}\left[\mathrm{N}(\mathrm{CN})_{2}\right](8.5 \mathrm{~g}, 0.05 \mathrm{~mol})$ in water $(20 \mathrm{ml})$ was stirred at $80^{\circ} \mathrm{C}$ for $1 \mathrm{~h}$, the mixture was filtered and the water removed under vacuum. The colourless liquid was then washed with diethyl ether $(3 \times 20 \mathrm{ml})$ and dried under vacuum for $24 \mathrm{~h}$, yield: $10.9 \mathrm{~g}, 82 \%$; ESIMS $\left(\mathrm{H}_{2} \mathrm{O}\right)$ : positive ion: 201 [C $\mathrm{C}_{4}$ Allylsul], negative ion: $66\left[\mathrm{~N}(\mathrm{CN})_{2}\right] .{ }^{1} \mathrm{H} \mathrm{NMR}\left(\mathrm{D}_{2} \mathrm{O}\right): \delta=5.98(\mathrm{~m}$, $1 \mathrm{H}), 5.61(\mathrm{~s}, 1 \mathrm{H}), 5.55(\mathrm{~s}, 1 \mathrm{H}), 3.84(\mathrm{~m}, 2 \mathrm{H}), 3.08$ (triple, $4 \mathrm{H}), 1.79$ (penta $4 \mathrm{H}) ; 1.51$ (penta $4 \mathrm{H}), 0.99$ (triple, $6 \mathrm{H}){ }^{13} \mathrm{C}$ NMR $\left(\mathrm{D}_{2} \mathrm{O}\right): \delta=127.8,123.4,118.3,40.7,37.9,25.6,21.2,12.74 ; \mathrm{IR}\left(\mathrm{cm}^{-1}\right): 2969$, 2950 ( $v_{\mathrm{C}-\mathrm{H}}$ alkane), 1638 ( $v_{\mathrm{C}=\mathrm{C}}$ alkene); Anal. Calcd for $\mathrm{C}_{14} \mathrm{H}_{25} \mathrm{~N}_{3} \mathrm{~S}(\%)$ : C, 62.88; H, 9.42; N, 15.71; Found: C, 62.88, H, 9.41, N, 15.80. 


\section{Synthesis of [Allylthio]I 7}

A solution of tetrahydrothiophene $(8.8 \mathrm{~g}, 0.10 \mathrm{~mol})$ and allyl iodide $(16.8 \mathrm{~g}, 0.10 \mathrm{~mol})$ in acetone $(20 \mathrm{ml})$ was stirred at r.t. for $24 \mathrm{~h}$, during which time a yellow-brown viscous phase formed. The solvent was removed and the liquid was washed with diethyl ether $(3 \times 30 \mathrm{ml})$ and dried under vacuum for $24 \mathrm{~h}$, yield: $24.3 \mathrm{~g}, 95 \%$; ESI-MS $\left(\mathrm{H}_{2} \mathrm{O}\right)$ : positive ion: 129 [Allylthiol], negative ion: 127 [I]. ${ }^{1} \mathrm{H}$ NMR $\left(\mathrm{D}_{2} \mathrm{O}\right): \delta=5.89(\mathrm{~m}, 1 \mathrm{H}), 5.85(\mathrm{~s}, 1 \mathrm{H}), 5.57(\mathrm{~s}, 1 \mathrm{H}), 4.42(\mathrm{~m}, 2 \mathrm{H}), 3.80$ (triple, 2H), 3.64 (triple, 2H), 2.46 (triple $4 \mathrm{H}) ;{ }^{13} \mathrm{C}$ NMR $\left(\mathrm{D}_{2} \mathrm{O}\right): \delta=127.7,125.1,44.7,43.3,29.4$; IR $\left(\mathrm{cm}^{-1}\right): 3182,2939\left(\mathrm{v}_{\mathrm{C}-\mathrm{H}}\right.$ alkane), 1655 ( $v_{\mathrm{C}=\mathrm{C}}$ alkene); Anal. Calcd for $\mathrm{C}_{7} \mathrm{H}_{13} \mathrm{IS}(\%)$ : C, 32.82; H, 5.12; Found: C, 32.82; H, 5.14.

\section{Synthesis of [Allylthiol] $\left[\mathrm{Tf}_{2} \mathrm{~N}\right] \mathrm{8}$}

A solution of $7(12.8 \mathrm{~g}, 0.05 \mathrm{~mol})$ and $\mathrm{Li}\left[\mathrm{Tf}_{2} \mathrm{~N}\right](13.4 \mathrm{~g}, 0.05 \mathrm{~mol})$ in water $(20 \mathrm{ml})$ was stirred at r.t. for $1 \mathrm{~h}$, during which time a second liquid phase formed. The water was removed under vacuum and the ionic liquid phase was washed with water $(3 \times 20 \mathrm{ml})$ and dried under vacuum for $24 \mathrm{~h}$, yield: $15 \mathrm{~g}$, 74\%; ESI-MS (Methanol): positive ion: 129 [Allylthio], negative ion: 280 [Tf $\left.{ }_{2} \mathrm{~N}\right] .{ }^{1} \mathrm{H} \mathrm{NMR}\left(\mathrm{D}_{2} \mathrm{O}\right): \delta=$ $5.90(\mathrm{~m}, 1 \mathrm{H}), 5.67(\mathrm{~s}, 1 \mathrm{H}), 5.59(\mathrm{~s}, 1 \mathrm{H}), 3.88(\mathrm{~m}, 2 \mathrm{H}), 3.50$ (triple, 2H), 3.33 (triple, 2H), 2.27 (triple $4 \mathrm{H}) ;{ }^{13} \mathrm{C}$ NMR $\left(\mathrm{D}_{2} \mathrm{O}\right): \delta=126.7,124.9,117.4,44.0,42.1,28.4 ; \mathrm{IR}\left(\mathrm{cm}^{-1}\right): 2954$ (v ${ }_{\mathrm{C}-\mathrm{H}}$ alkane $), 1638$ $\left(v_{\mathrm{C}=\mathrm{C}}\right.$ alkene); Anal. Calcd for $\mathrm{C}_{9} \mathrm{H}_{13} \mathrm{~F}_{6} \mathrm{NO}_{4} \mathrm{~S}_{3}(\%)$ : C, 26.40; H, 3.20; N, 3.42; Found: C, 26.42; H, $3.14 ; \mathrm{N}, 3.44$.

\section{Synthesis of [Allylthio $]\left[\mathrm{N}(\mathrm{CN})_{2}\right] 9$}

A solution of $7(12.8 \mathrm{~g}, 0.05 \mathrm{~mol})$ and $\mathrm{Ag}\left[\mathrm{N}(\mathrm{CN})_{2}\right](8.5 \mathrm{~g}, 0.05 \mathrm{~mol})$ in water $(20 \mathrm{ml})$ was stirred at $80^{\circ} \mathrm{C}$ for $1 \mathrm{~h}$, then the mixture was filtered and the water removed under vacuum at $80^{\circ} \mathrm{C}$. The colourless liquid was then washed with diethyl ether $(3 \times 20 \mathrm{ml})$ and dried under vacuum for $24 \mathrm{~h}$, yield: 8.7 g, 89\%; ESI-MS (Ethanol): positive ion: 129 [Allylthio], negative ion: $64\left[\mathrm{~N}(\mathrm{CN})_{2}\right] .{ }^{1} \mathrm{H}$ NMR $\left(\mathrm{D}_{2} \mathrm{O}\right): \delta=6.21(\mathrm{~m}, 1 \mathrm{H}), 5.98(\mathrm{~s}, 1 \mathrm{H}), 5.94(\mathrm{~s}, 1 \mathrm{H}), 4.21(\mathrm{~m}, 2 \mathrm{H}), 3.82$ (triple, 2H), 3.71 (triple, 2H), 2.62 (triple, $4 \mathrm{H}) ;{ }^{13} \mathrm{C}$ NMR $\left(\mathrm{D}_{2} \mathrm{O}\right): \delta=127.6,125.2,119.8,44.7,42.6,29.0$; IR $\left(\mathrm{cm}^{-1}\right): 3488$, 2978, 2950 ( $v_{\mathrm{C}-\mathrm{H}}$ alkane), $1636\left(v_{\mathrm{C}=\mathrm{C}}\right.$ alkene); Anal. Calcd for $\mathrm{C}_{9} \mathrm{H}_{13} \mathrm{~N}_{3} \mathrm{~S}(\%): \mathrm{C}, 55.35 ; \mathrm{H}, 6.71 ; \mathrm{N}$, 21.52; Found: C, 55.38; H, 6.74; N, 21.58.

\section{Structural characterisation in the solid state}

Data was collected on a four-circle Kappa goniometer equipped with an Oxford Diffraction KM4 Sapphire CCD at 140(2) K and data reduction was performed using CrysAlis RED [29]. Structure solution was carried out using SiR92 and refined by full-matrix least-squares refinement (against $F^{2}$ ) using the SHELXTL software [30]. All non-hydrogen atoms were refined anisotropically while hydrogen atoms were placed in their geometrically generated positions and refined using the riding model. Disorder for the aliphatic groups was modeled using the PART function implemented in 
SHELXTL. Empirical absorption corrections (DELABS) were applied [ 31 ], and graphical representations of the structures were made with Diamond [32]. CCDC 641220 contains the supplementary crystallographic data for this paper. These data can be obtained free of charge from The Cambridge Crystallographic Data Centre via www.ccdc.cam.ac.uk/data_request/cif.

\section{Crystal Data for 1}

$\left[\mathrm{C}_{7} \mathrm{H}_{15} \mathrm{~S}\right] \mathrm{Br}, \mathrm{M}_{\mathrm{w}}=211.16$, crystal system = trigonal, $\mathrm{a}=17.8173(8) \AA, \mathrm{b}=17.8173(8) \AA$, $\mathrm{c}=21.4885(14) \AA, \alpha=90^{\circ}, \beta=90^{\circ}, \gamma=120^{\circ}, \mathrm{V}=5907.7(5) \AA^{3}, \mathrm{~T}=140(2) \mathrm{K}$, space group $=\mathrm{R} 3 \mathrm{c}, \mathrm{Z}$ $=24,($ Mo-K $)=0.71073 \AA, 11152$ reflections collected, 2305 independent reflections, $\mathrm{R}_{\text {int }}=0.0466$, $\mathrm{R} 1[\mathrm{I}>2(\mathrm{I})]=0.0311, \mathrm{wR} 2($ all data $)=0.0734$.

\section{Acknowledgements}

We thank the Swiss National Science Foundation and the EPFL for the financial support, and Dr. Rosario Scopelliti and Dr Euro Solari for collecting the X-ray diffraction data.

\section{References and Notes}

1. For leading reviews see: (a) Welton, T. Room-Temperature Ionic Liquids. Solvents for Synthesis and Catalysis. Chem. Rev., 1999, 99, 2071-2084. (b) Wasserscheid, P.; Keim, W. Ionic Liquids New "Solutions" for Transition Metal Catalysis. Angew. Chem. Int. Ed. Engl. 2000, 39, 3772-3789. (c) Gordon, C. M. New developments in catalysis using ionic liquids. App. Catal. A: General 2001, 222, 101-117. (c) Olivier-Bourbigou, H.; Magna, L. Ionic liquids: perspectives for organic and catalytic reactions. J. Mol. Catal.: A, 2002, 182-183, 419-437. (d) Zhao. D.; Wu, M. ; Kou, Y. ; Min, E. Ionic liquids: applications in catalysis. Catal. Today, 2002, 74, 157-189. (e) Dupont, J.; de Souza, R. F.; Suarez, P. A. Z. Ionic Liquid (Molten Salt) Phase Organometallic Catalysis. Chem. Rev., 2002, 102, 3667-3692.

2. Anderson, J. L.; Armstrong, D. W.; Wei, G.-T. Ionic Liquids in Analytical Chemistry. Anal. Chem. 2006, 78, 2893-2902.

3. (a) Davis. Jr, J. H. Task-Specific Ionic Liquids. Chem. Lett. 2004, 33, 1072-1077. (b) Fei, Z.; Geldbach, T. J.; Zhao, D.; Dyson, P. J. From Dysfunction to Bis-function: On the Design and Applications of Functionalised Ionic Liquids. Chem. Eur. J. 2006, 12, 2122-2130.

4. (a) Xue, H.; Verma, R.; Shreeve, J. M. Review of ionic liquids with fluorine-containing anions. $J$. Fluorine Chem. 2006, 127, 159-176. (b) Li, X.; Zhao, D.; Fei, Z.; Wang, L. Applications of functionalized ionic liquids. Science in China, Series B, 2006, 49, 385-401. 
5. For example see, (a) Chen, W.; Liu, F.; Synthesis and characterization of oligomeric and polymeric silver-imidazol-2-ylidene iodide complexes. J. Organomet. Chem. 2003, 673, 5-12. (b) Schottenberger, H.; Wurst, K.; Horvath, U. E. I.; Cronje, S.; Lukasser, J.; Polin, J.; McKenzie, J. M.; Raubenheimer, H. G. Synthesis and characterisation of organometallic imidazolium compounds that include a new organometallic Ionic liquid. Dalton Trans. 2003, 4275-4281. (c) Fei, Z.; Zhao, D.; Scopelliti, R.; Dyson, P. J. Organometallic Complexes Derived from AlkyneFunctionalized Imidazolium Salts. Organometallics, 2004, 23, 1622-1628. (d) Zhao, D.; Fei, Z.; Ang, W. H.; Scopelliti, R.; Dyson, P. J. Thiocyanate Functionalised Ionic Liquids: Synthesis, Characterisation and Reactivity. Eur. J. Inorg. Chem, 2007, 279-284. (e) Fei, Z.; Ang, W. H.; Geldbach, T. J.; Scopelliti, R.; Dyson, P. J. Ionic Solid-State Dimers and Polymers Derived from Imidazolium Dicarboxylic Acids. Chem. Eur. J., 2006, 12, 4014-4020. (f) Cole, A. C.; Jensen, J. L.; Ntai, I.; Tran, K. L. T.; Weaver, K. J.; Forbes, D. C.; Davis Jr. J. H. Novel Brønsted Acidic Ionic Liquids and Their Use as Dual Solvent-Catalysts. J. Am. Chem. Soc. 2002, 124, 5962-5963. (g) Holbrey, J. D.; Reichert, W. M.; Tkatchenko, I.; Bouajila, E.; Walter, O.; Tommasi, I. 1,3Dimethylimidazolium-2-carboxylate: the unexpected synthesis of an ionic liquid precursor and carbene- $\mathrm{CO}_{2}$ adduct. Chem. Commun. 2003, 28-29. (h) Fei, Z.; Zhao, D.; Geldbach, T. J.; Scopelliti, R.; Dyson, P. J. Brønsted Acidic Ionic Liquids and Their Zwitterions: Synthesis, Characterization and $p$ Ka Determination. Chem. Eur. J. 2004, 10, 4886-4893. (i) Itoh, H.; Naka, K.; Chujo, Y. Synthesis of Gold Nanoparticles Modified with Ionic Liquid Based on the Imidazolium Cation. $J$. Am. Chem. Soc. 2004, 126, 3026-3027. (j) Visser, A. E.; Swatloski, R. P.; Reichert, W. M.; Mayton, R.; Sheff, S.; Wierzbicki, A.; Davis Jr., J. H.; Rogers, R. D. Task-specific ionic liquids for the extraction of metal ions from aqueous solutions. Chem. Commun. 2001, 135-136. (k) Gao, Y.; Twamley, B.; Shreeve, J. M. The First (Ferrocenylmethyl)imidazolium and (Ferrocenylmethyl)triazolium Room Temperature Ionic Liquids. Inorg. Chem., 2004, 43, 34063412. (1) Fei, Z.; Zhao, D.; Pieraccini, D.; Ang, W. H.; Geldbach, T. J.; Scopelliti, R.; Chiappe, C.; Dyson, P. J. Development of Nitrile-Functionalized Ionic Liquids for C-C Coupling Reactions: Implication of Carbene and Nanoparticle Catalysts. Organometallics, 2007, 26, 1588-1598.

6. (a) Zhou, Z.-B.; Matsumoto, H.; Tatsumi, K. Low-Melting, Low-Viscous, Hydrophobic Ionic Liquids: 1-Alkyl(Alkyl Ether)-3-methylimidazolium Perfluoroalkyltrifluoroborate. Chem. Eur. J. 2005, 10, 6581-6891. (b) Zhou, Z.-B.; Matsumoto, H.; Tatsumi, K. Low-Melting, Low-Viscous, Hydrophobic Ionic Liquids: Aliphatic Quaternary Ammonium Salts with Perfluoroalkyltrifluoroborates. Chem. Eur. J. 2005, 11, 752-766. 
7. (a) Zhao, D.; Fei, Z.; Geldbach, T. J.; Scopelliti, R.; Dyson, P. J. Nitrile-Functionalized Pyridinium Ionic Liquids: Synthesis, Characterization, and Their Application in Carbon-Carbon Coupling Reactions. J. Am. Chem. Soc. 2004, 126, 15876-15882. (b) Singh, R. P.; Winter, R. W.; Gard, G. L.; Gao, Y.; Shreeve, J. M. Quaternary Salts Containing the Pentafluorosulfanyl ( $\left.\mathrm{SF}_{5}\right)$ Group. Inorg. Chem. 2003, 42, 6142-6146.

8. Xue, H.; Twamley, B.; Shreeve, J. M. The First 1-Alkyl-3-perfluoroalkyl-4,5- dimethyl-1,2,4triazolium Salts. J. Org. Chem. 2004, 69, 1397-1400.

9. Kim, J.; Singh, R. P.; Shreeve, J. M. Low Melting Inorganic Salts of Alkyl-, Fluoroalkyl-, Alkyl Ether-, and Fluoroalkyl Ether-Substituted Oxazolidine and Morpholine. Inorg. Chem. 2004, 43, 2960-2966.

10. Gao, Y.; Shreeve, J. M. Quaternization of pyrazine, pyridazine, and pyrimidine with alkyl and polyfluoroalkyl halides: Formation of low melting salts. Synthesis 2004, 1072-1082.

11. (a) Brown, R. J. C.; Dyson, P. J.; Ellis, D. J.; Welton, T. 1-Butyl-3-methylimidazolium cobalt tetracarbonyl $[\mathrm{bmim}]\left[\mathrm{Co}(\mathrm{CO})_{4}\right]$ : a catalytically active organometallic ionic liquid. Chem. Commun, 2001, 1862-1863. (b) Zhao, D.; Fei, Z.; Ohlin, C. A.; Laurenczy, G.; Dyson, P. J. Dualfunctionalised ionic liquids: synthesis and characterisation of imidazolium salts with a nitrilefunctionalised anion. Chem. Commun. 2004, 2500-2501. (c) Zhou, Z.-B.; Matsumoto, H.; Tatsumi, K. Low-Viscous, Low-Melting, Hydrophobic Ionic Liquids: 1-Alkyl-3-methylimidazolium Trifluoromethyltrifluoroborate. Chem. Lett. 2004, 33, 680-681.

12. Paulsson, H.; Hagfeldt, A.; Kloo, L. Molten and Solid Trialkylsulfonium Iodides and Their Polyiodides as Electrolytes in Dye-Sensitized Nanocrystalline Solar Cells. J. Phys. Chem. B 2003, $107,13665-13670$.

13. (a) Wang, P.; Wenger, B.; Humphry-Baker, R.; Moser, J.-E.; Teuscher, J.; Kantlehner, W.; Mezger, J.; Stoyanov, E. V.; Zakeeruddin, S. M.; Grätzel, M. Charge Separation and Efficient Light Energy Conversion in Sensitized Mesoscopic Solar Cells Based on Binary Ionic Liquids. J. Am. Chem. Soc. 2005, 127, 6850-6856. (b) Wang, P.; Zakeeruddin, S. M.; Compte P.; Exnar, I.; Grätzel, M. Gelation of Ionic Liquid-Based Electrolytes with Silica Nanoparticles for Quasi-Solid-State DyeSensitized Solar Cells. J. Am. Chem. Soc. 2003, 125, 1166-1167. (c) Mazille, F.; Fei, Z.; Kuang, D.; Zhao, D.; Zakeeruddin, S. M.; Grätzel, M.; Dyson, P. J. Influence of Ionic Liquids Bearing Functional Groups in Dye-Sensitized Solar Cells. Inorg. Chem. 2006, 45, 1585-1590.

14. (a) Paulsson, H.; Berggrund, M.; Svantesson, E.; Hagfeldt, A.; Kloo, L.; Molten and solid metaliodide-doped trialkylsulphonium iodides and polyiodides as electrolytes in dye-sensitized 
nanocrystalline solar cells. Sol. Energy Mater. Sol. Cells 2004, 82, 345-360. (b) Paulsson, H.; Kloo, L.; Hagfeldt, A.; Boschloo, G. Electron transport and recombination in dye-sensitized solar cells with ionic liquid electrolytes. J. Electroanalyt. Chem. 2006, 586, 56-61.

15. (a) Wang, Y.; Zhang, W.; Colandrea, V. J.; Jimenez, L. S. Reactivity and rearrangements of dialkyl- and diarylvinylsulfonium salts with indole-2- and pyrrole-2-carboxaldehydes. Tetrahedron, 1999, 55, 10659-10672. (b) Xu, Y.; Fletcher, M.; Dolbier Jr., W. R. J. Org. Chem, 2000, 65, 3460. (c) Aggarwal, V. K.; Fang, G. Y.; Schmidt. A. T. Synthesis and Applications of Chiral Organoboranes Generated from Sulfonium Ylides. J. Am. Chem. Soc., 2005, 127, 1642-1643.

16. Gerhard, D.; Alpaslan, S. C.; Gores, H. J.; Uerdingen, M.; Wasserscheid, P. Trialkylsulfonium dicyanamides - a new family of ionic liquids with very low viscosities. Chem. Commun. 2005, 5080-5082.

17. Kuang, D.; Wang, P.; Ito, S.; Zakeeruddin, S. M.; Grätzel, M. Stable Mesoscopic Dye-Sensitized Solar Cells Based on Tetracyanoborate Ionic Liquid Electrolyte. J. Amer. Chem. Soc., 2006, 128, 7732-7733.

18. Kim, H. S.; Kim, Y. J.; Lee, H.; Park, K. Y.; Lee, C.; Chin, C. S. Ionic Liquids Containing Anionic Selenium Species: Applications for the Oxidative Carbonylation of Aniline. Angew. Chem. Int. Ed. 2002, 41, 4300-4303.

19. Zhou, Z.-B.; Takeda, M.; Ue, M. New hydrophobic ionic liquids based on perfluoroalkyltrifluoroborate anions. J. Flourine Chem. 2004, 125, 471-476.

20. (a) Zhao, D.; Fei, Z.; Geldbach, T. J.; Scopelliti, R.; Laurenczy, G.; Dyson, P. J. AllylFunctionalised Ionic Liquids: Synthesis, Characterisation, and Reactivity. Helv. Chim. Acta 2005, 88, 665-675. (b) Zhang, H.; Wu, J.; Zhang, J.; He, J. 1-Allyl-3-methylimidazolium Chloride Room Temperature Ionic Liquid: A New and Powerful Nonderivatizing Solvent for Cellulose. Macromolecules, 2005, 38, 8272-8277.

21. Fei, Z.; Kuang, D.; Zhao, D.; Klein, C.; Ang, W. H.; Zakeeruddin, S. M.; Grätzel, M.; Dyson, P. J. A Supercooled Imidazolium Iodide Ionic Liquid as a Low-Viscosity Electrolyte for Dye-Sensitized Solar Cells. Inorg. Chem. 2006, 45, 10407-10409.

22. Hoffman, J. L. Ion chromatographic analysis of the purity and synthesis of sulfonium and selenonium ions. Journal of Chromatography, 1991, 588, 211-216.

23. Dyson, P. J.; McIndoe, J. S.; Zhao, D. Direct analysis of catalysts immobilised in ionic liquids using electrospray ionisation ion trap mass spectrometry. Chem. Commun. 2003, 508-509. 
24. (a) Busi, S.; Lahtinen, M.; Ropponen, J.; Valkonen, J.; Rissanen, K. Synthesis, characterization and thermal properties of new aromatic quaternary ammonium bromides: precursors for ionic liquids and complexation studies. J. Solid State Chem. 2004, 177, 3757-3767. (b) Ralle, M.; Bryan, J. C.; Habenschuss, A.; Wunderlich, B. Low-Temperature Phase of Tetraethylammonium Bromide. Acta Crystallogr., Sect. C: 1997, C53, 488-490.

25. Mizumo, T.; Marwanta, E.; Matsumi, N.; Ohno, H. Allylimidazolium Halides as Novel Room Temperature Ionic Liquids. Chem. Lett. 2004, 33, 1360-1361.

26. (a) Eike, D. M.; Brennecke, J. F.; Maginn, E. J. Predicting melting points of quaternary ammonium ionic liquids. Green Chem. 2003, 5, 323-328. (b) Krossing, I.; Slattery, J. M.; Daguenet, C.; Dyson, P. J.; Oleinikova, A.; Weingärtner, H. Why Are Ionic Liquids Liquid? A Simple Explanation Based on Lattice and Solvation Energies. J. Am. Chem. Soc., 2006, 128, 13427-13434. (c) Katsyuba, S. A.; Zvereva, E. E.; Vidiš, A.; Dyson, P. J. Application of Density Functional Theory and Vibrational Spectroscopy Toward the Rational Design of Ionic Liquids. J. Phys. Chem. A, 2007, 111, 352-370.

27. Zhou, Z.-B.; Matsumoto, H.; Tatsumi, K. Cyclic Quaternary Ammonium Ionic Liquids with Perfluoroalkyltrifluoroborates: Synthesis, Characterization, and Properties. Chem. Eur. J. 2006, 12, 2196-3312.

28. Dyson, P. J.; McIndoe, J. S. Analysis of organometallic compounds using ion trap mass spectrometry. Inorg. Chim. Acta. 2003, 354, 68-74.

29. Oxford Diffraction Ltd., Abingdon, Oxfordshire, UK, 2002

30. Sheldrick, G. M. University of Göttingen, Germany, 1997; Bruker AXS, Inc., Madison, Wisconsin, 53719, USA, 1997

31. Walker, N.; Stuart, D. An Empirical Method for Correcting Diffractometer Data for Absorption Effects. Acta Crystallogr. Sect A 1983, 39, 158-166.

32. Diamond 3.0a, Crystal Impact GbR, Bonn, Germany.

(C) 2007 by MDPI (http://www.mdpi.org). Reproduction is permitted for noncommercial purposes. 